\title{
Auriculocondylar syndrome
}

INSERM

\section{Source}

INSERM. (1999). Orphanet: an online rare disease and orphan drug data base.

Auriculocondylar syndrome. ORPHA:137888

Auriculo-condylar syndrome (ACS) presents with bilateral external ear malformations

('question mark' ears), mandibular condyle hypoplasia, microstomia, micrognathia,

microglossia and facial asymmetry. Additional manifestations include hypotonia, ptosis,

cleft palate, puffy cheeks, developmental delay, impaired hearing and respiratory distress. 